\title{
Recurrence of embolism post intravenous thrombolysis on an asymptomatic bilateral carotid stenosis
}

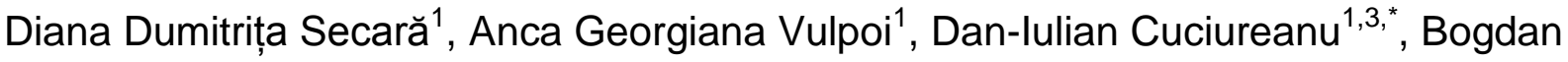 \\ Secară ${ }^{1}$, Alexandru Chiriac ${ }^{1}$, Radu Vulpoi ${ }^{2}$, Tudor Cuciureanu ${ }^{2}$ \\ 1"Prof. Dr. N. Oblu" Clinical Emergency Hospital, lasi, Romania; ªstroenterology Department, \\ "Grigore T. Popa" University of Medicine and Pharmacy lasi, Romania; ${ }^{3}$ Neurology Department, \\ "Grigore T. Popa" University of Medicine and Pharmacy lasi, Romania
}

\begin{abstract}
Atherosclerosis is the most common cause of stroke and patients with stroke due to large-vessel atherosclerosis are at the highest risk of recurrent stroke. Carotid endarterectomy (CEA) is a secondary prevention method for reducing the risk of stroke, but endovascular therapy has developed rapidly in recent years. The long-term therapeutic efficacy of endovascular stenting for carotid artery stenosis has been shown not to be inferior to CEA. We present the case of a 61-year-old female, smoker, dyslipidemic, hypertensive, therapeutically neglected, who suddenly developed right hemiplegia accompanied by central facial palsy and motor aphasia. The inclusion criteria allowed the initiation of intravenous thrombolytic therapy. At 2 and 24 hours after thrombolysis the evolution was favorable with the almost complete recovery of motor and speech production deficits. At 48 hours after thrombolysis, the patient experienced rapid decompensation and worsening, with recurrence of hemiplegia on the same side and motor aphasia. Cervical echoDoppler and cerebro-cervical CT angiography (CTA) revealed a complete right internal carotid artery (ICA) and quantified the degree of left ICA stenosis asymptomatic up to that time. The case was a challenge in terms of subsequent secondary prevention. Although most studies dealt with endarterectomy as a treatment for tight carotid stenosis, in the here reported case this was not possible, so we had to resort to angioplasty with placement of a self-expandable stent in the left ICA.
\end{abstract}

Keywords: thrombolysis, asymptomatic stenosis, endarterectomy, self-expandable stent

\section{Introduction}

Stroke (ischemic or hemorrhagic) is the main cause of long-term disability and the second leading cause of mortality in the world. Asymptomatic carotid stenosis over $60 \%$ is responsible for $1-2 \%$ of stroke cases/year, the risk rising to $3-4 \% /$ year when stenosis, active plaque or contra lateral carotid occlusion are present. Atherosclerosis with ICA stenosis accounts for approximately $20 \%$ of ischemic

Received: October 2017; Accepted after review: December 2017; Published: December 2017.

${ }^{*}$ Corresponding author: Assoc. Prof. Dan-Iulian Cuciureanu MD, PhD, Department of Neurology, "Grigore T. Popa" University of Medicine and Pharmacy 16 Universitatii Street, lasi 700115, Romania.

E-mail: cuciureanu57@gmail.com strokes [1].

Patients with stroke due to large-vessel atherosclerosis are at the highest risk of recurrent stroke, $37 \%$ of recurrences occurring within the first 7 days. Early carotid revascularization performed after intravenous thrombolysis could increase the efficacy of stroke prevention strategy [2].

Endarterectomy remains the first-line treatment for symptomatic patients with stenosis of $50 \%$ to $99 \%$. Large randomized trials have shown moderate benefit of endarterectomy versus drug treatment for asymptomatic stenosis over $60 \%$. Angioplasty and stenting is reserved for symptomatic patients with stenosis of $50 \%$ to $99 \%$ or at high risk for open surgery because of anatomical or clinical reasons. The decision to perform revascularization in patients with carotid artery 
stenosis and the choice of treatment depends on several criteria including: severity of internal carotid artery stenosis, age, sex, comorbidities, life expectancy and complication rate $[1,3,4]$.

\section{Case Report}

A 61-year-old female patient, residing in an urban area, presented to the Emergency Department of the "Nicolae Oblu" Emergency Hospital, with a complete motor impairment of the right limbs, accompanied by speech and language disorders and facial asymmetry, with the onset of symptoms one hour before hospital presentation. From her medical history, we found out that she was recently diagnosed with grade II hypertension, with high added risk, the patient having dyslipidemia and being a heavy smoker.

General physical examination revealed no significant changes. Objective neurological examination revealed right hemiplegia $(\mathrm{MRC}=0 / 5)$, with central facial paresis and global aphasia (NIHSS $=16$ points). Investigations were performed to assess the opportunity of initiating intravenous thrombolysis. Laboratory tests were within normal ranges; the native cranio-cerebral CT scan did not detect signs of acute ischemic or hemorrhagic stroke, arteriovenous malformations or subarachnoid hemorrhage (Figure 1).

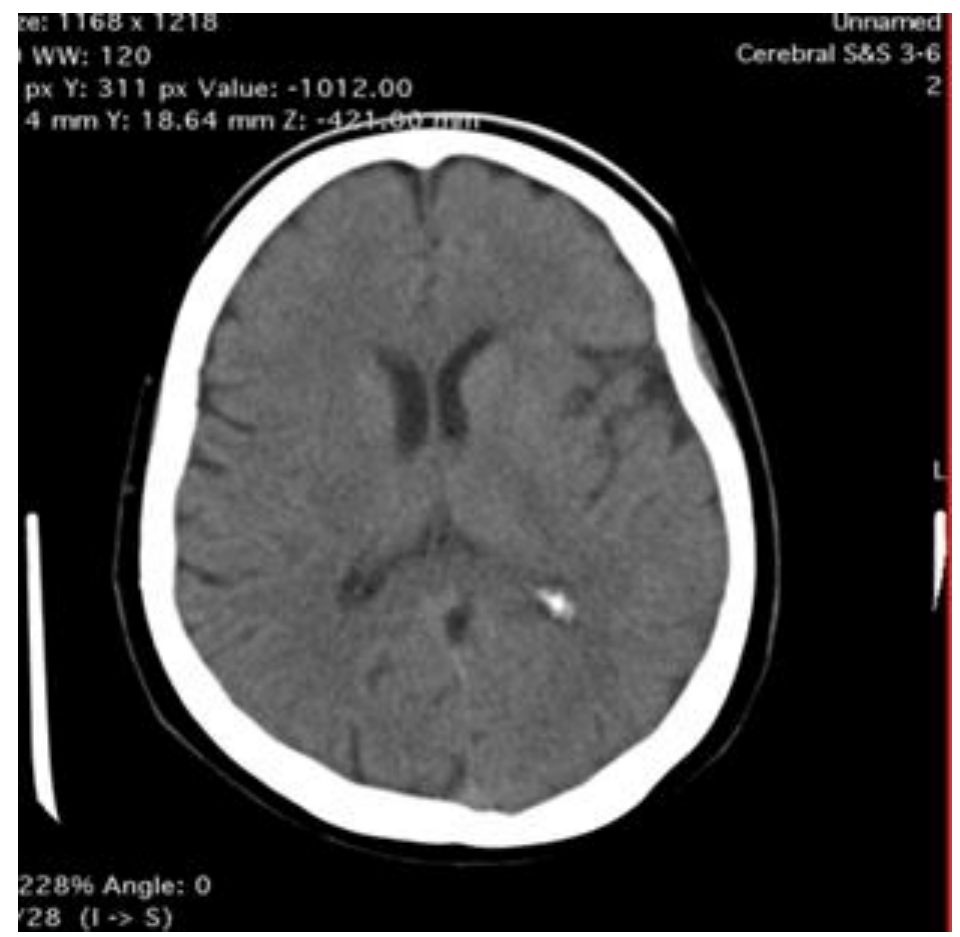

Fig. 1. Native cranio-cerebral CT image (ischemic lesions, cerebral atherosclerosis, leukoaraiosis, age-related changes)

With the written consent of the family and in the absence of contraindications, intravenous thrombolysis at a dose of $72 \mathrm{mg}$ (7.2 $\mathrm{mg}$ intravenous bolus, then $62.8 \mathrm{mg}$ via intravenous perfusion, over 1 hour) was initiated, with improvement of the motor deficit, facial asymmetry and speech and language disorders at the end of perfusion, symptoms corresponding to a NIHSS score of 5 points (of the 16 points before iv. administration of Actylise).

Within the first 24 hours, the outcome was favorable, the native craniocerebral CT scan, demonstrating a hypodense area suggestive of a recent ischemic stroke at the left basal nucleus and partially at the head of the caudate nucleus (Figure 2). 


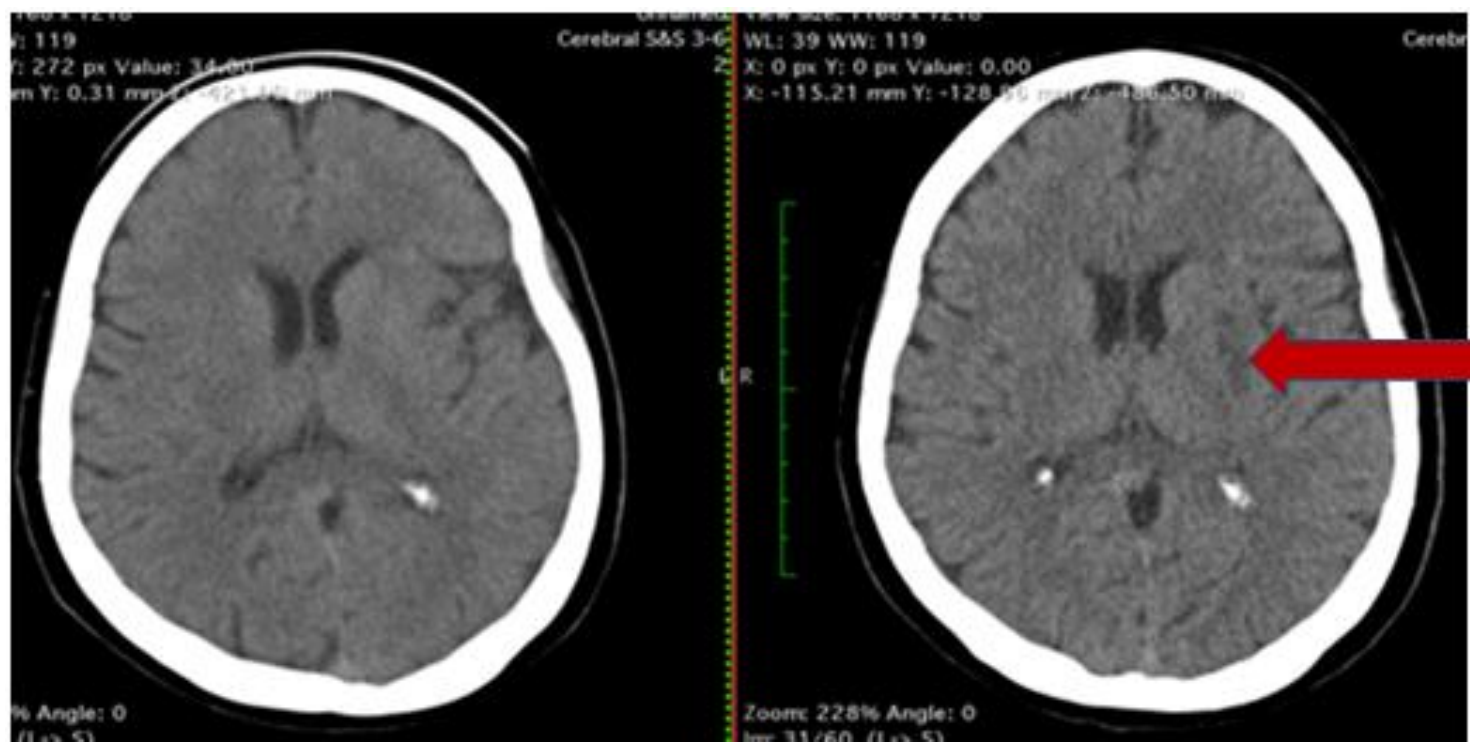

Fig. 2. Cranio-cerebral CT images at admission (left) and 24 hours (right), where the hypodense area in the left basal nuclei and head of the caudate nucleus can be seen

In this situation the patient received antiplatelet aggregation, $4 \times 75 \mathrm{mg}$ tablets/day, and a high statin dose. In the absence of a specific contraindication, patients with acute ischemic stroke should be treated with a platelet antiaggregant within 48 hours of symptom onset (delayed for 24 hours in those treated with IV-tP). Treating patients who have experienced a noncardioembolic stroke with a high-potency statin reduces the risk of recurrent events [5].

At 36 hours after the presentation, the patient presented again a complete motor deficit with global aphasia and facial asymmetry, probably due to early mobilization without medical recommendation; the patient was reassessed with imaging methods and the enlargement of the ischemic area was noted (Figure 3).

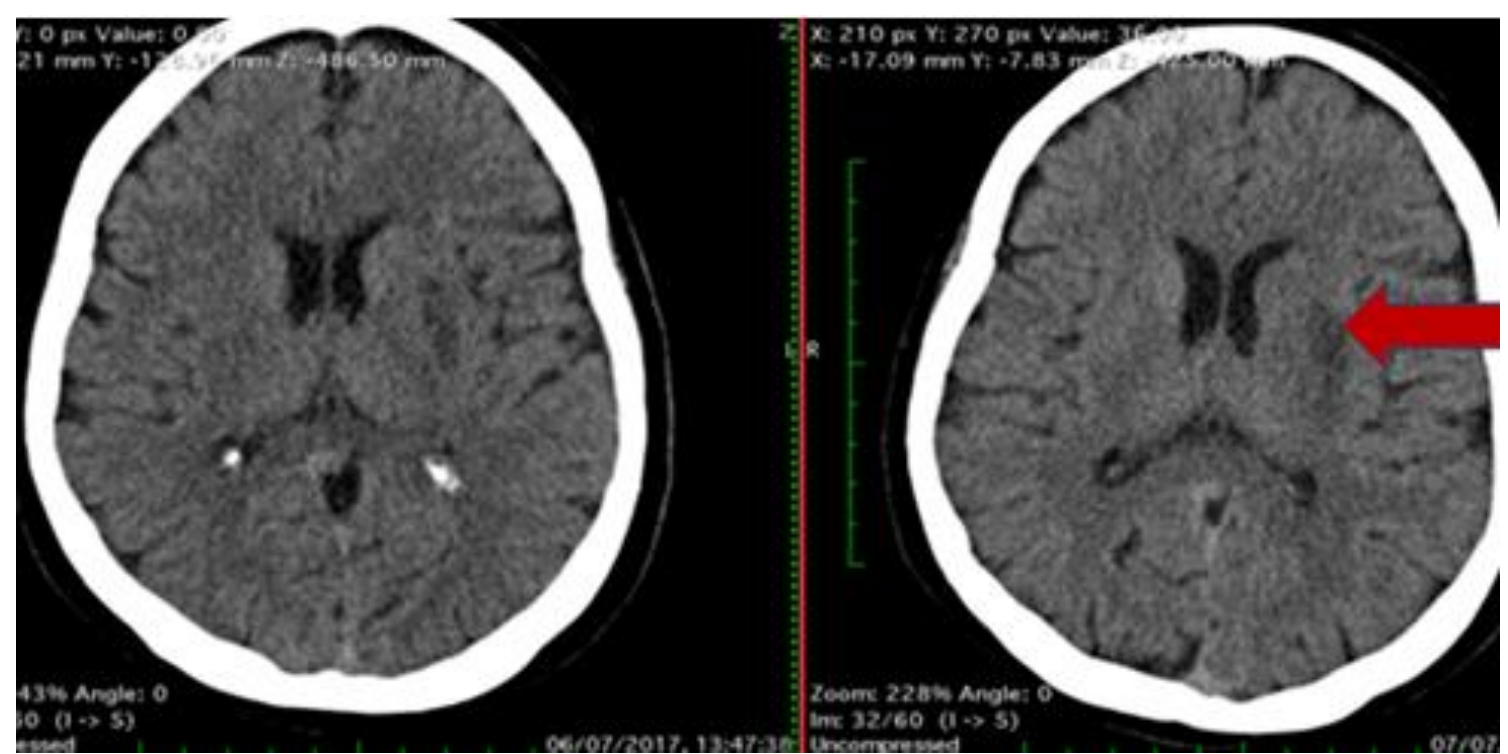

Fig. 3. Native cranio-cerebral CT images before (left) and after the enlargement of the ischemic area (right) 
Subsequently, laboratory investigations were performed, in order to determine the cause of the ischemic stroke and of its recurrence. Cardiac examination revealed rhythmic cardiac sounds and electrocardiogram showed no significant alterations except for left ventricular hypertrophy. Later, the cardiac investigations were continued at the Institute of
Cardiovascular Diseases. Echo-Doppler examination of the cervical vessel detected numerous inhomogeneous calcareous plaques at the bifurcation of the left carotid artery, which cause severe stenosis, and also in the internal carotid artery (Figure 4); on the right side the complete occlusion of the right internal carotid artery was noticed.

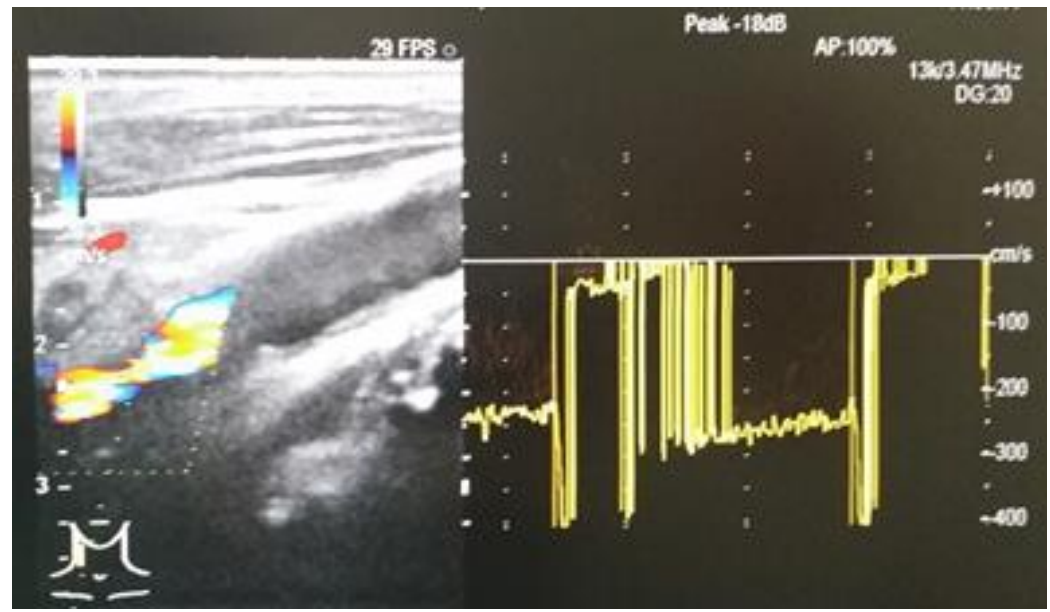

Fig. 4. Echo-Doppler of cervical vessels: severe stenosis of the left internal carotid artery (> 85\%)

Investigations were continued with CT angiography of cerebral and cervical arteries that confirmed the lesions previously described by echo-Doppler, showing a 50\% left common carotid artery stenosis at its bifurcation, an $85 \%$ left internal carotid artery stenosis (Figure 5), as well as complete occlusion of the right internal carotid artery (Figure 6).

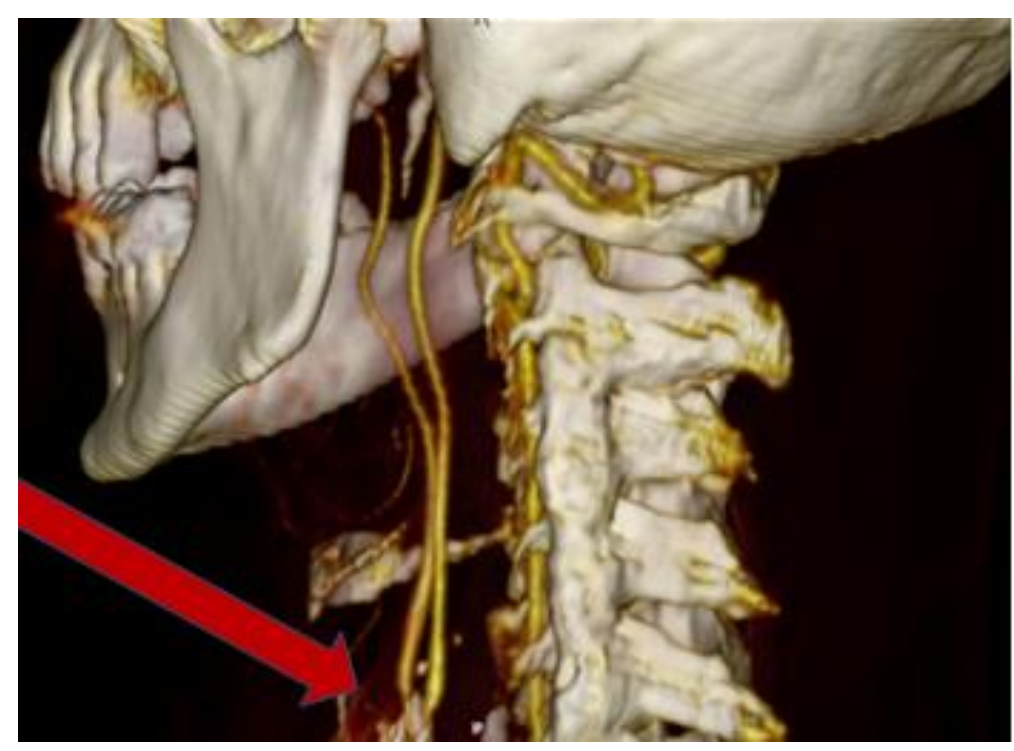

Fig. 5. CT angiography of the cervical vessels which demonstrated a 50\% left common carotid artery stenosis at its bifurcation and an $85 \%$ left internal carotid artery sever stenosis 


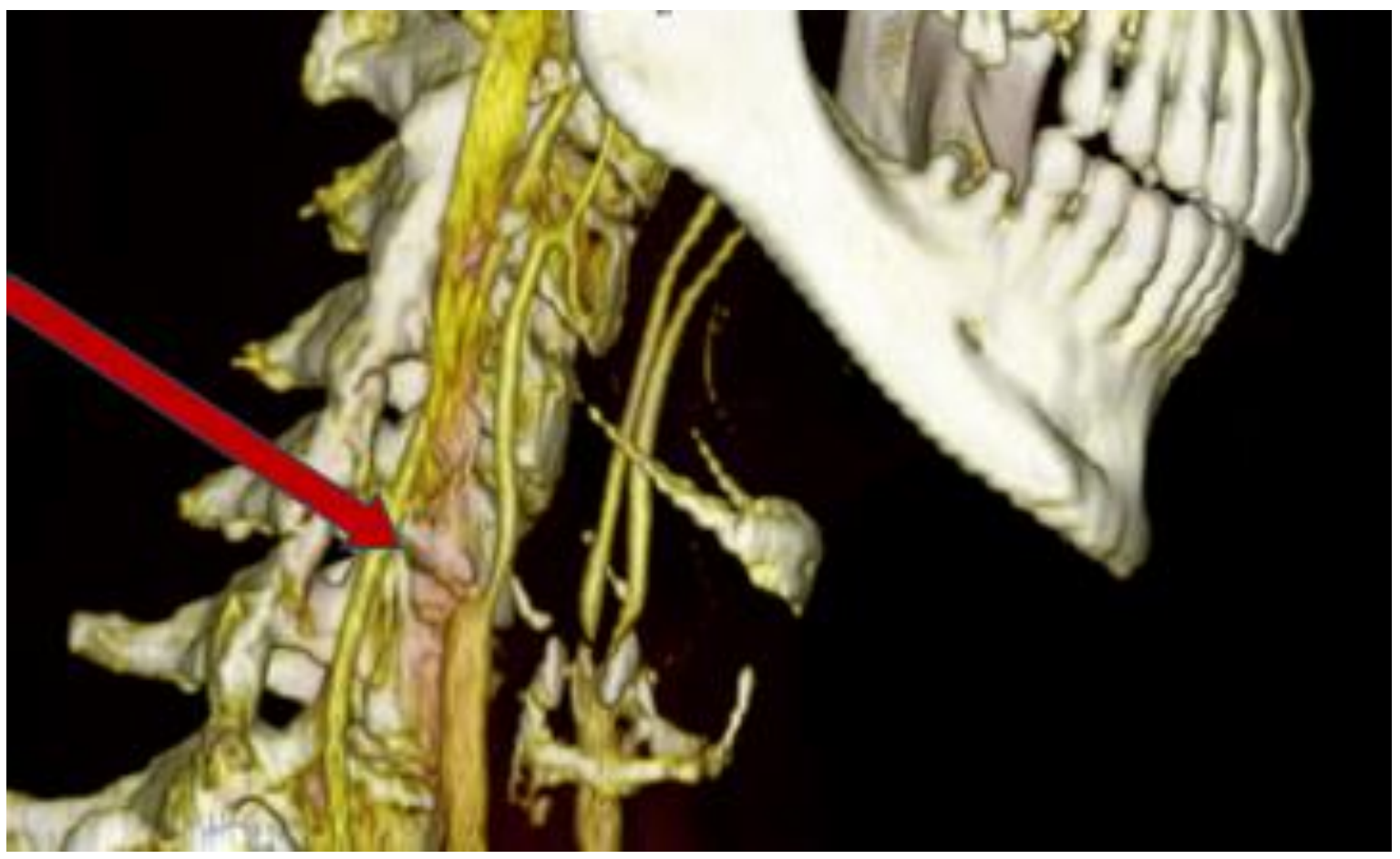

Fig. 6. CT angiography of cervical vessels showing complete occlusion of the right internal carotid artery

The subsequent course was slowly favorable, with improvement of the asymmetric motor deficit (MRC 2/5 upper right limb, MRC $3 / 5$ lower right limb), and persistence of speech disorder (motor aphasia). Referral of the patient for neuromotor rehabilitation, endovascular investigation and surgical or endovascular treatment was required.

The particularity of this case required a multidisciplinary approach - a team composed of neurologist, radiologist, neurosurgeon and interventional cardiologist. The patient was referred to the neurosurgery clinic for endovascular exploration of carotid artery and if required the initiation of endovascular or surgical treatment (Figure 7).

Next, we had to choose a type of treatment for severe symptomatic carotid stenosis. Because of the contralateral internal carotid occlusion, endarterectomy was not possible, although patients with symptomatic internal carotid artery stenosis of $70-99 \%$ are recommended to undergo carotid endarterectomy for the prevention of ischemic stroke recurrence (Class I indication).

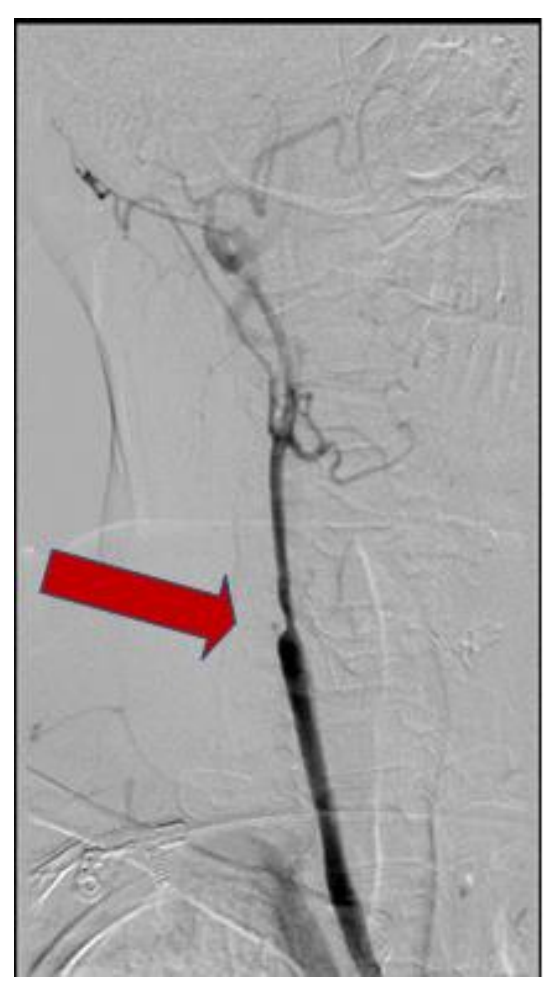

Fig. 7. Seldinger angiography of the cervical vessels showing complete occlusion of the right internal carotid artery 


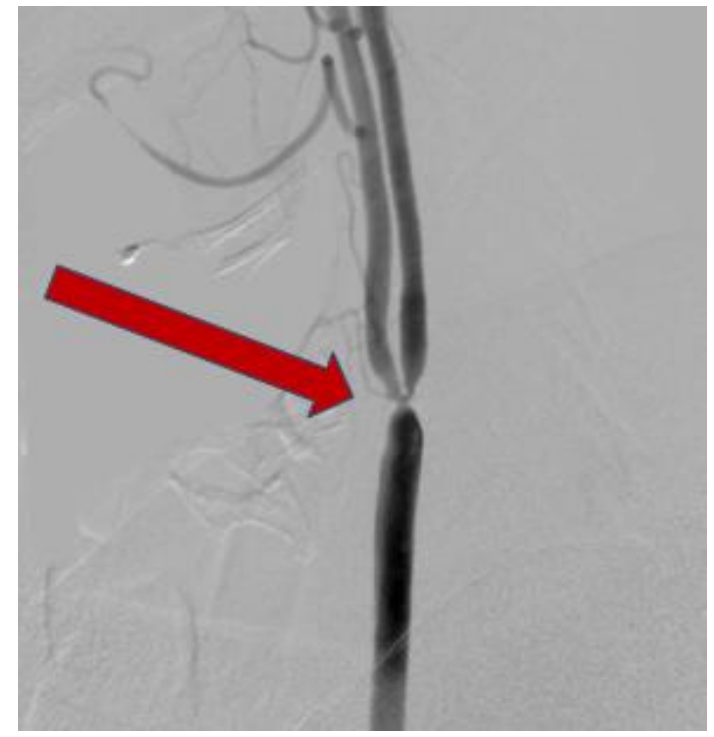

Fig. 8. Seldinger angiography of the cervical vessels showing severe stenosis at the bifurcation of the left internal carotid artery

We opted for percutaneous angioplasty and stenting, but, given the anatomic particularity of patient's vascular system detachment of the left common carotid artery from the aortic arch in oblique position), stenting in the neurosurgery clinic was impossible, so the patient was referred to interventional cardiology (Figure 9). The procedure was performed by right femoral approach intubating the left common carotid artery with a guide catheter, followed by the implantation of a self-expandable stent. Angiographic control revealed normal intracerebral flow with residual stenosis of $0 \%$ (Figure 10).

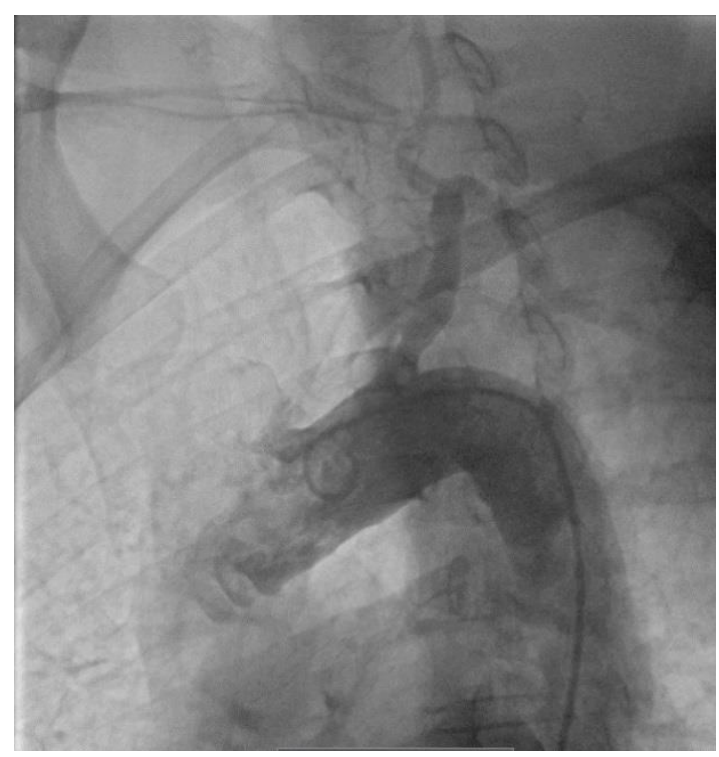

Fig. 9. Seldinger angiography of the detachment of the left common carotid artery from the aortic arch in oblique position
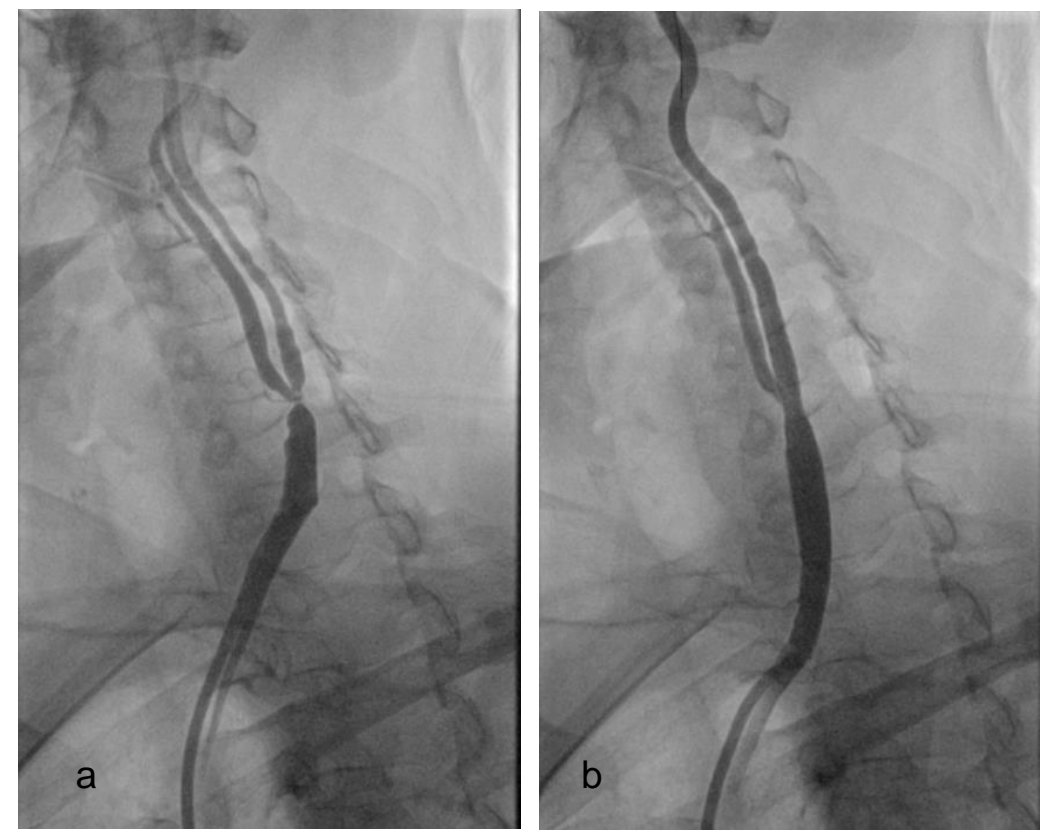

Fig. 10. Seldinger angiography of the left internal carotid artery before (a) and after (b) the implantation of a selfexpandable stent. Angiographic control revealed normal intracerebral flow with residual stenosis of $0 \%$. 
After stenting, in addition to the control of cardiovascular risk factors, treatment consisted in the administration of statin and a combination of two antiaggregant drugs (clopidogrel and aspirin) for at least 6 months. Two randomized studies compared the use of aspirin alone vs. aspirin + clopidogrel after stenting, studies ended prematurely due to the increased risk of stent thrombosis and cerebrovascular events in patients exclusively treated with aspirin.

The course was favorable, with improvement of neurological symptoms up to a Barthel score of 12 at 3 months and a Rankin score of 3.

\section{Discussions}

A global analysis of the ESCT and NASCET studies (5893 patients with 33,000 patient-years of follow-up) demonstrated that carotid revascularization should be performed rapidly in symptomatic patients with transient ischemic attack (TIA) or mild stroke, showing that endarterectomy is effective in preventing recurrent stroke in patients with symptomatic stenosis of ICA. Delayed surgery increases the chance of recurrent stroke and decreases the benefits of surgery, which is why endarterectomy is recommended within the first 1 to 2 weeks of stroke, if the cerebral infarction area is $1-2 \mathrm{~cm}$ and the neurological symptoms are mild, and within 2-4 weeks if the ischemic area is wider due to the high operative risk of reperfusion injury and hemorrhage in the cerebral infarction after reopening of the stenotic carotid artery $[1,3]$.

The first study to assess the safety of patients who after intravenous thrombolysis underwent early revascularization suggests that carotid stenosis can be considered a safe alternative to endarterectomy following rtPA administration in the prevention of stroke recurrence. The study included 145 patients who underwent i.v. thrombolysis, 6 of whom had after thrombolysis carotid stenosis $>/<70 \%$ and ulcerated/unstable atherosclerotic plaques. Stenting was performed very early but after the first 24 hours, and follow up at 3 , 6 , and 12 months revealed stent patency and favorable course, except for one patient who suffered another stroke after four months, associated with an episode of paroxysmal fibrillation, and not due to the instability of atherosclerotic plaques [2].

A study (Heo SH et al.) conducted in 2017 compared restenosis rates after carotid endarterectomy (CEA) and carotid stenting (CAS) in unselected patients and the incidence of major adverse clinical events (stroke, TIA, myocardial infarction, or death) and found that: of the 1184 patients divided into 2 groups: 654 CEA and 530 CAS, the CAS group experienced a higher incidence of major adverse events ( $7.5 \%$ vs. $2.4 \%$ ), but with a lower incidence of procedure-related complications (1.5\% vs. $5.3 \%)$; restenosis rates were higher after CAS than after CEA (1.5\% vs. $1.0 \%$ at 12 months and $5.4 \%$ vs. $1.2 \%$ at 24 months, respectively), demonstrating that CEA remains the main method of choice in treating carotid stenosis due to fewer adverse effects [6].

Related to the use of stenting, six large clinical trials (enrolling $>300$ patients) comparing CEA with CAS were performed. CAVATAS 94, EVA-3S95, ICSS 96, and SPACE 97 studies enrolled only symptomatic patients. The SAPPHIRE 98,99 and CREST 79 studies included both symptomatic and asymptomatic patients at high or conventional risk for surgery. The results of these studies do not clearly demonstrate the superiority of endarterectomy or of stenting, the decision belonging to the treating physician, adapted to the particularity of the case [2].

\section{Conclusions}

The reported case illustrates the good results of vascular recanalization after intravenous thrombolysis followed by a recurrence being based of the same pathogenic mechanism - thromboembolism originating in asymptomatic active atherosclerotic plaques.

This case report represents an acute diagnosis and also a therapeutic challenge because of a multitude of particularities of patient's cerebral blood vessels (path, old arterial occlusion, stenosis), raising the 
question of possible different immediate attitudes after thrombolysis.

Complex secondary prophylaxis by multidisciplinary effort suggests the need for an individualized, revisited, emergency,

\section{References}

1. Tendera M, Aboyans V, Bartelink ML, et al. ESC Guidelines on the diagnosis and treatment of peripheral artery diseases. $R$ J Cardiol 2012; (3):240-247.

2. Sallustio F, Koch G, Rocco A, et al. Safety of early carotid artery stenting after systemic thrombolysis: a single center experience. Stroke Res Treat 2012; 2012:904575.

3. Markus H, Pereira A, Cloud G. Secondary prevention of stroke. In Oxford Specialist Handbooks in Neurology-Stroke Medicine, Second Edition, Oxford University Press, 2017; (10):302-332. multidisciplinary approach to acute stroke management.

\section{Conflict of interest}

Authors declare no conflict of interest.

4. Flumignan $C D Q$, Flumignan RLG, Navarro TP. Extracranial carotid stenosis: evidence based review. Rev Col Bras Cir 2017; 44(3):293-301.

5. Larry B. Goldstein modern medical management of acute ischemic stroke. Methodist Debakey Cardiovasc J 2014; 10(2):99-104.

6. Heo SH, Yoon KW, Woo SY, et al. Comparison of early outcomes and restenosis rate between carotid endarterectomy and carotid artery stenting using propensity score matching analysis. Eur J Vasc Endovasc Surg 2017; 54(5):573-578. 